Boulevard, Los Angeles, California 90027, USA. e-mail:dwarburton@chla.usc.edu

1. Metzger, R. J., Klein, O. D., Martin, G. R. \& Krasnow, M. A Nature 453, 745-750 (2008).

2. Maillieux, A. A. et al. Mech. Dev. 102, 81-94 (2001).

3. Unbekandt, M. et al. Mech. Dev. 125, 314-324 (2008).
4. Affolter, M. et al. Dev. Cell 4, 11-18 (2003).

5. Tefft, D. J. et al. Curr. Biol. 9, 219-222 (1999).

6. Warburton, D. et al. Pediatr. Res. 57, 26R-37R (2005).

7. Cardoso, W. V. \& Lü, J. Development 133, 1611-1624 (2006).

8. De Robertis, E. M. Cell 132, 185-195 (2008)

9. Centanin, L. et al. Dev. Cell 14, 547-558 (2008).

10. Del Moral, P.-M. et al. Dev. Biol. 290, 177-188 (2006).

\title{
PHYSICAL CHEMISTRY
}

\section{Charge states in transition}

Raffaele Resta

\section{Transition metals come in different oxidation states with different electric charges. So at least we are told at school. Detailed calculations lead to a heretical conclusion - those variable charge states are a myth.}

The idea of electric charge 'belonging' to a given atom or ion has been a central one ever since Michael Faraday, studying the use of electric current to decompose ionic compounds, published his laws of electrolysis in 1832. The charge that passes between two electrodes can be measured; it is always an integer multiple of the basic electronic charge; and it is proportional to the number of atoms exchanged. At the root of this effect is a basic quantization phenomenon ${ }^{1}$ of the kind that still exercises physicists investigating, for example, the quantum Hall effect ${ }^{2}$. Both electrolysis and the quantum Hall effect deal with charges in transit. But when instead we address the static charges belonging to atoms in compounds, no such quantization theorem can be invoked. Such charges seem arbitrarily defined: the charges one gets are non-integer, and cannot be reliably measured.

So what is the relationship between these static charges and 'oxidation states' - the formal states of integer charge that today underlie our ideas of electrolysis and much else in chemistry? On page 763 of this issue, Raebiger, Lany and Zunger ${ }^{3}$ address this question in transition metals, which are notable for having more than one stable oxidation state. Their answer? There is no connection: a change in the oxidation state of a transition-metal atom occurs without noticeable change in its net physical charge. The authors provide a consummately elegant explanation for this finding as the consequence of a feedback mechanism that leads the charge on the transition-metal atom to regulate itself. Furthermore, they show that this mechanism is present in both semiconducting and ionic host materials.

The concept of atomic oxidation states was introduced by the American chemist Wendell Latimer in 1938. It is undoubtedly useful for bookkeeping in chemical reactions involving molecules, crystals or liquids. An agreed set of rules ${ }^{4}$ is used to attribute an integer oxidation state to all the atoms of a given compound. This oxidation state corresponds to the charge (in units of electronic charge) that an atom would have if its bonds were entirely ionic. In reality, when two nearest-neighbour atoms are of different species, their bond is neither entirely ionic nor entirely covalent. It is thus misleading, and a little dangerous, to attribute any physical meaning to the Coulomb energy ascribed to an atomic point charge by its oxidation state.

Even in the archetypal ionic crystal, sodium chloride, a change in oxidation state of 1 exaggerates the physical charge transfer between atoms of the two species, which is between around 0.7 and 0.8 (ref. 1). Further away from the extreme ionic limit, where the character of bonds between different atomic species is strongly covalent, characterized by the sharing rather than the swapping of electrons, this disparity becomes even greater. For transitionmetal atoms, covalence is important even in bonds to significantly electronegative species such as oxygen, where ionic bonding would be expected to dominate ${ }^{5}$.

Raebiger et al. ${ }^{3}$ perform a series of firstprinciple calculations of the charges on single transition-metal atoms embedded in a large semiconducting or ionic matrix. They consider different occupation states for transition-metal energy levels that sit in the band gap of the surrounding matrix structure - that is, in an energy range where the matrix itself has no allowed energy levels. This configuration allows the oxidation state of the transition metal to be varied while changing (almost) nothing else.

Because the bonds formed by transitionmetal atoms are always partly covalent, the energy levels of the transition metal and its partner in the bonding are strongly hybridized. The hybridization of transition-metal and oxygen energy levels has been thoroughly investigated. In the class of compound oxides known as perovskites, hybridization is largely responsible for their characteristic 'ferroelectricity' - their spontaneous polarization even in the absence of an electric field ${ }^{5,6}$.

Quite generally, covalence creates what amounts to a constructive interference between the quantum wavefunctions of the partner atoms. In Raebiger and colleagues' simulations, interference between the wavefunctions of the transition metal and the surrounding atoms contributes to a net atomic charge around the transition-metal atom. The authors explain their core finding - that the net physical charge belonging to a transition-metal atom is essentially independent of its oxidation state - with the following feedback mechanism. Suppose we wish to add an electron to the transition-metal atom, and thus change its oxidation state; then, unavoidably, the hybridization of its energy levels with those of its partner atom changes. This change always has the effect of displacing some negative charge away from the transition metal; the extra charge flows away and is spread over a large region, and the net physical charge belonging to the transition-metal atom within the structure stays essentially constant.

The charge transfers associated with changes in oxidation states have often been considered as a first approximation to the physical, ionic charge of a transition-metal atom, with covalent hybridization accounting for just a small correction to it. Raebiger et al. show ${ }^{3}$ otherwise: charge self-regulation, rather than charge transfer, is the appropriate model.

Different oxidation states of the same transition-metal atom have distinct 'ionic radii' and distinct signatures, such as shifts in the X-ray light emitted from their core levels. These features must now be reconsidered in terms of the degree of hybridization, not the net physical charge at the transition-metal site. Knowledge of the electron density alone is not enough to address such subtleties; the full quantum-mechanical density matrix must be analysed instead. Tools devised to this end include charge-and-bondorder analysis and the electron localization function ${ }^{7,8}$. By applying such tools, we can hope to achieve an understanding of transitionmetal bonding in different oxidation states that is finally on a sound theoretical footing.

Raffaele Resta is in the Dipartimento di Fisica Teorica, Università di Trieste, strada Costiera 11, 34014 Trieste, Italy.

e-mail: resta@democritos.it

1. Pendry, J. B. \& Hodges, C. H. J. Phys. C 17, 1269-1279 (1984).

2. Thouless, D. J. Topological Quantum Numbers in Nonrelativistic Physics (World Scientific, Singapore, 1998)

3. Raebiger, H., Lany, S. \& Zunger, A. Nature 453, 763-766 (2008)

4. http://goldbook.iupac.org/004365.html

5. Cohen, R. E. Nature 358, 136-138 (1992)

6. Rabe, K. M., Ahn, Ch. H. \& Triscone, J.-M. Physics of Ferroelectrics: A Modern Perspective (Springer, Berlin, 2007).

7. Becke, A. D. \& Edgecombe, K. E. J. Chem. Phys. 92, 5397-5403 (1990)

8. Silvi, B. \& Savin, A. Nature 371, 683-685 (1994). 